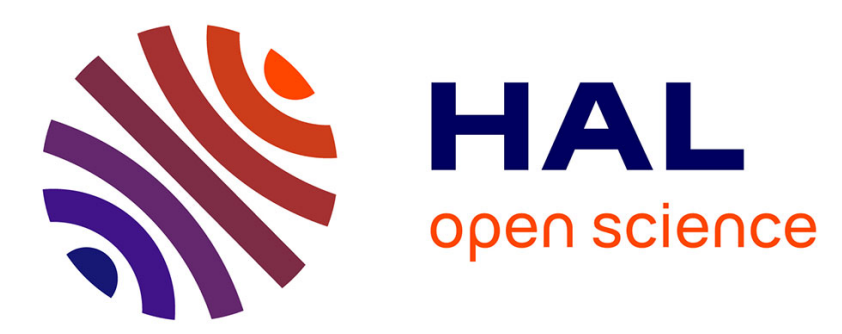

\title{
Détermination de la taille et de la concentration de cristallites dans une couche amorphe par mesure de conductivité
}

\author{
A. Gheorghiu, S. Squelard, K. Zellama, P. Germain, J.C. Bourgoin
}

\section{To cite this version:}

A. Gheorghiu, S. Squelard, K. Zellama, P. Germain, J.C. Bourgoin. Détermination de la taille et de la concentration de cristallites dans une couche amorphe par mesure de conductivité. Revue de Physique Appliquée, 1977, 12 (5), pp.721-723. 10.1051/rphysap:01977001205072100 . jpa-00244233

HAL Id: jpa-00244233

https://hal.science/jpa-00244233

Submitted on 1 Jan 1977

HAL is a multi-disciplinary open access archive for the deposit and dissemination of scientific research documents, whether they are published or not. The documents may come from teaching and research institutions in France or abroad, or from public or private research centers.
L'archive ouverte pluridisciplinaire HAL, est destinée au dépôt et à la diffusion de documents scientifiques de niveau recherche, publiés ou non, émanant des établissements d'enseignement et de recherche français ou étrangers, des laboratoires publics ou privés. 


\title{
DÉTERMINATION DE LA TAILLE ET DE LA CONCENTRATION DE CRISTALLITES DANS UNE COUCHE AMORPHE PAR MESURE DE CONDUCTIVITÉ
}

\author{
A. GHEORGHIU \\ Laboratoire d'Optique des Solides (*), Université Paris 6, Tour 13, \\ 4, place Jussieu, 75230 Paris, France
}

et

\author{
S. SQUELARD, K. ZELLAMA, P. GERMAIN, J. C. BOURGOIN
}

Groupe de Physique des Solides de l'E. N. S. (**), Université Paris 7, Tour 23, 2, place Jussieu, 75331 Paris, France

\begin{abstract}
Résumé. - On rappelle comment la fraction cristallisée peut être obtenue à partir d'une mesure de conductivité. On montre brièvement comment la vitesse de croissance, la taille et la concentration des cristallites peuvent être déterminés en étudiant la cinétique de cristallisation, suivant que celle-ci est induite en surface ou en volume, dans le cas où il n'y a pas nucléation homogène. On présente des résultats expérimentaux pour des couches de germanium amorphe.
\end{abstract}

\begin{abstract}
We recall the way a cristalline fraction can be obtained using conductivity measurements. We show briefly how the growth rate of crystallization, the size and the concentration of crystallites can be determined from the study o the crystallization kinetics, depending whether the crystallization is surface induced or bulk induced, in case where no homogeneous nucleation occurs. We present experimental results for amorphous germanium layers.
\end{abstract}

1. Introduction. - La détermination de la taille et de la concentration des cristallites présents dans une couche amorphe peut être faite à partir de l'étude de la cinétique de cristallisation. En effet l'évolution en temps de la fraction cristallisée dépend de la vitesse de croissance des cristallites $\left(v_{\mathrm{g}}\right)$, du taux de nucléation $(n)$ et des conditions initiales : concentration $p$ et taille de cristallites (rayon $a$ ).

Le but de cette communication est de montrer comment on peut obtenir $v_{g}, a$ et $p$ à partir de la cinétique de cristallisation obtenues par mesure de conductivité lorsque la cristallisation se fait sans nucléation homogène $(n=0)$. Cette étude ayant déjà fait l'objet de publications $[1,2]$ on ne fera ici que rappeler brièvement le principe de la détermination de $p, a$ et $v_{\mathrm{g}}$ à partir de la fraction cristallisée (1 ${ }^{\text {re }}$ partie) et la relation entre la fraction cristallisée et la conductivité ( $2^{\mathrm{e}}$ partie) ; on présentera ensuite ( $3^{\mathrm{e}}$ partie) une série de résultats qui complètent les résultats décrits dans les références [1] et [2].

2. Cinétique de cristallisation. - Lorsque la cris-

(*) Laboratoire associé au C. N. R.S.

(**) Travail subventionné en partie par la délégation générale à la Recherche Scientifique et Technique. tallisation se produit sans nucléation homogène, la fraction cristallisée s'écrit [2] :

$$
x_{2}(t)=1-\exp -\left[\frac{p \pi}{s}\left(a+v_{\mathrm{g}} t\right)^{2}\right]
$$

( $s$ : surface de la couche) pour des temps supérieurs à un temps caractéristique $\tau$. Dans le cas où la cristallisation est induite à la surface et que $a$ est supérieur à l'épaisseur $e$ de la couche amorphe ce temps $\tau$ correspond au temps pendant lequel la fraction cristallisée reste linéaire avec le temps :

$$
x_{2}(t)=p \pi a^{2} v_{\mathrm{g}} t / \mathrm{Se} .
$$

En d'autres termes la fraction cristallisée est donnée par l'expression (1) lorsque la taille des cristallites est supérieure à l'épaisseur de la couche. On voit immédiatement que la comparaison entre les variations expérimentaleset théoriques de $x_{2}$ (du second degré en temps) permettent d'obtenir $p$ et $a$ lorsque $v_{g}$ est connue. La détermination, de $v_{\mathrm{g}}$ peut se faire dans un régime ou la cristallisation se produit à partir de la surface ; en effet le temps $\tau$ pendant lequel la fraction cristallisée est linéaire dans le temps est tel que :

$$
v_{\mathrm{g}} \tau=e .
$$


3. Relation entre fonction cristallisée et conductivité. - La conductivité d'une couche amorphe présentant des cristallites est la conductivité moyenne $\sigma_{\mathrm{m}}$ d'un mélange de deux phases réparties aléatoirement (amorphe : 1 et cristalline : 2) qui peut être calculée [3] à partir des conductivités de ces deux phrases $\left(\sigma_{1}\right.$ et $\left.x_{2}\right)$ et des fractions volumiques correspondantes $\left(x_{1}\right.$ et $\left.x_{2}\right)$. On peut donc obtenir [2] $x_{2}$ à partir de $\sigma_{\mathrm{m}}$ lorsque $\sigma_{1}$ et $\sigma_{2}$ sont connues :

$\ln \left(\frac{1}{1-x_{2}}\right)=\ln \left[1-\left(\frac{\sigma_{1}-\sigma_{m}}{\sigma_{1}+2 \sigma_{m}}\right) /\left(\frac{\sigma_{2}-\sigma_{m}}{\sigma_{2}+2 \sigma_{m}}\right)\right]$.

Les courbes donnant $x_{2}$ et $\ln \left[1 /\left(1-x_{2}\right)\right]$ en fonction de $\sigma_{\mathrm{m}} / \sigma_{1}$ sont données dans la référence 2 dans le cas du germanium (pour lequel $\sigma_{1} / \sigma_{2} \simeq 20$ à la température où sont effectuées les mesures).

Dans le cas où la cristallisation est induite à la surface ou a montré [1] que la conductance de la couche est :

$$
\Sigma=\Sigma_{\alpha}\left[1+\left(\frac{\sigma_{\mathrm{m}}}{\sigma_{1}}-1\right) v_{\mathrm{g}} \frac{t}{e}\right]
$$

( $\Sigma_{\alpha}$ conductance de la couche totalement amorphe) pour $t \leqslant \tau$.

4. Résultats expérimentaux. - Les conditions expérimentales du dépôt des couches de germanium sont décrites en détail dans la référence [2].

L'étude de la cinétique de cristallisation de couches pour laquelle la cristallisation est induite à partir de la surface nous a permis de mesurer $v_{\mathrm{g}}$ en fonction de la température [1]. Les résultats, résumés sur la figure 1 ,

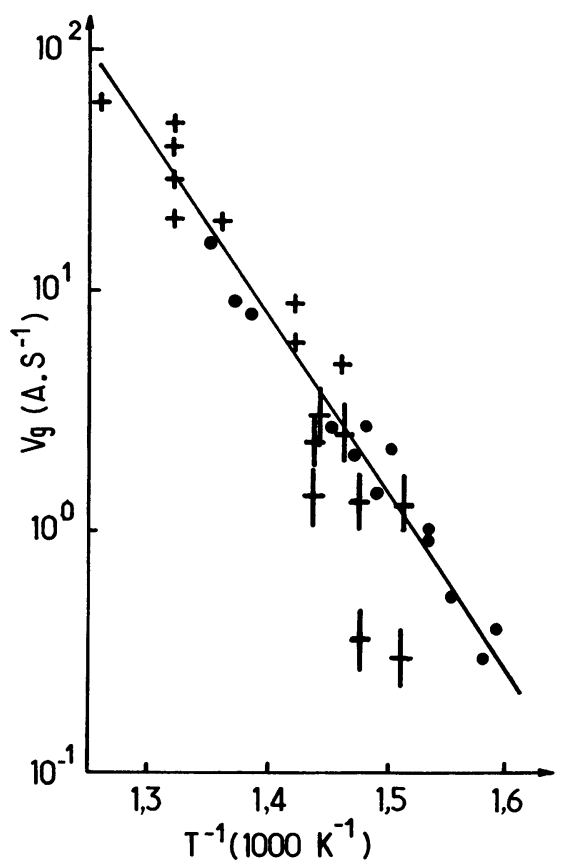

FIG. 1. - Variation de la vitesse de croissance avec $T^{-1}$ : + résultats de Barna et al. [4], - résultats présentés dans les références [1] et [2]; + échantillons des séries Ge XIV, XV et XVI. sont en bon accord avec les résultats obtenus par Barma et al. [4]. en microscope électronique.

En pratique il est apparu difficile d'obtenir de façon reproductible des échantillons de germanium qui cristallisent à partir de l'interface substrat-amorphe. On a donc été amené à fabriquer des échantillons formés d'une couche amorphe déposé sur une couche cristalline.

Ces échantillons sont obtenus de la façon suivante : on dépose d'abord une couche mince de germanium à haute température $\left(\sim 340^{\circ} \mathrm{C}\right)$ de façon à ce qu'elle soit cristallisée à la fin du dépôt ; on diminue alors la température du substrat $\left(\sim 30^{\circ} \mathrm{C}\right)$ et on dépose une couche amorphe sur cette couche polycristalline. Les conditions expérimentales sont résumées dans le tableau I.

\section{TABLEAU I}

\section{Conditions expérimentales de dépôt}

\begin{tabular}{lcccccccc}
\multicolumn{1}{c}{ Série } & $T_{1}$ & $T_{2}$ & $p_{1}$ & $p_{2}$ & $\theta$ & $e$ & $R_{1}$ & $R_{2}$ \\
- & - & - & - & - & - & - & - & - \\
Ge XIV & 340 & 30 & 4 & 2 & 55 & 0,5 & 25 & 4 \\
Ge XV & 340 & 30 & 3 & 1 & 55 & 1,62 & 1,7 & 4 \\
Ge XVI & 340 & 30 & 3 & 1,5 & 45 & 3,24 & 1,8 & 4
\end{tabular}

indice 1 : première évaporation ;

indice 2 : deuxième évaporation ;

$T$ température de substrat $\left({ }^{\circ} \mathrm{C}\right)$ lors de l'évaporation ;

$p$ pression résiduelle dans l'enceinte ;

$\theta$ intervalle de temps (min) entre les deux évaporations ;

$e$ épaisseur totale $(\mu \mathrm{m})$ de la couche mesurée par méthode interférentielle ;

$R$ résistance $(\Omega)$ de la couche $\left(\times 10^{6}\right)$ à température ambiante.

Les variations de $\Sigma(t) / \Sigma_{\alpha}$ en fonction du temps mesurées à différentes températures, sont données dans les figures 2 à 4 . Les valeurs de $v_{\mathrm{g}}$ que l'on en déduit son reportées sur la figure 1. Les écarts observés entre ces résultats et les résultats obtenus précédemment peuvent être attribués à une possible contamination à l'interface polycristal-amorphe.

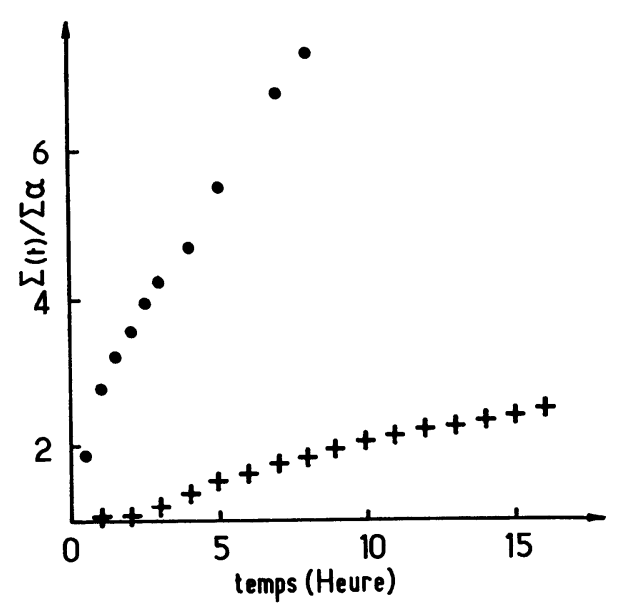

Fig. 2. - Variation de la quantité $\Sigma(t) / \Sigma_{\alpha}$ à $390^{\circ} \mathrm{C}$ et à $420^{\circ} \mathrm{C}$ (échantillons de la série Ge XIV). 


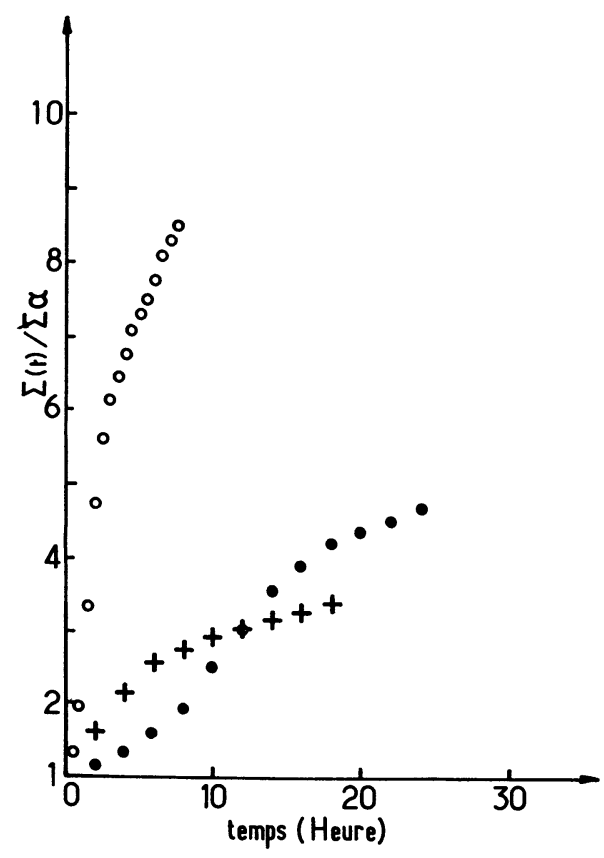

FIG. 3. - Variation de la quantité $\Sigma(t) / \Sigma_{\alpha}$ à $410^{\circ} \mathrm{C}, 390^{\circ} \mathrm{C}$ et $420^{\circ} \mathrm{C}$ (échantillons de la série Ge XVI).

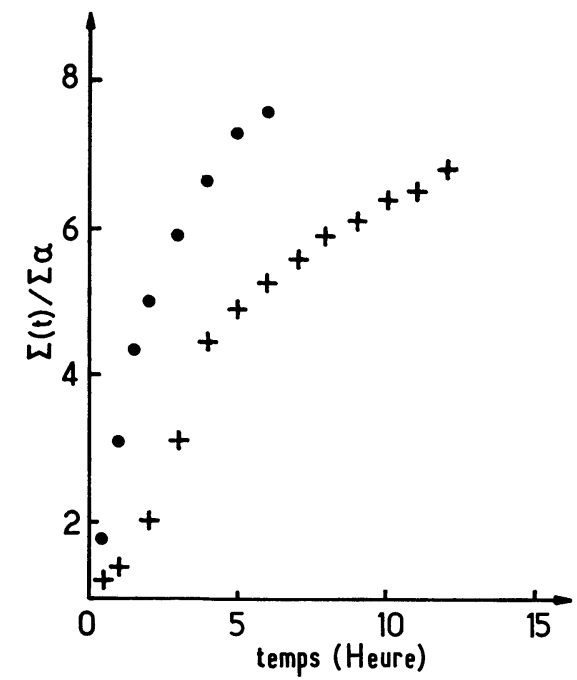

Fig. 4. - Variation de la quantité $\Sigma(t) / \Sigma_{\alpha}$ à $405^{\circ} \mathrm{C}$ et $420^{\circ} \mathrm{C}$ (échantillons de la série Ge XV).

La figure 5 donne la variation de la fraction cristallisée avec le temps, à différentes températures, dans le cas où la cristallisation est induite en volume. L'ajus- tement des points expérimentaux avec la relation du second degré (1) permet de déterminer des couples de valeurs $p$ et a à chaque température (qui correspondent

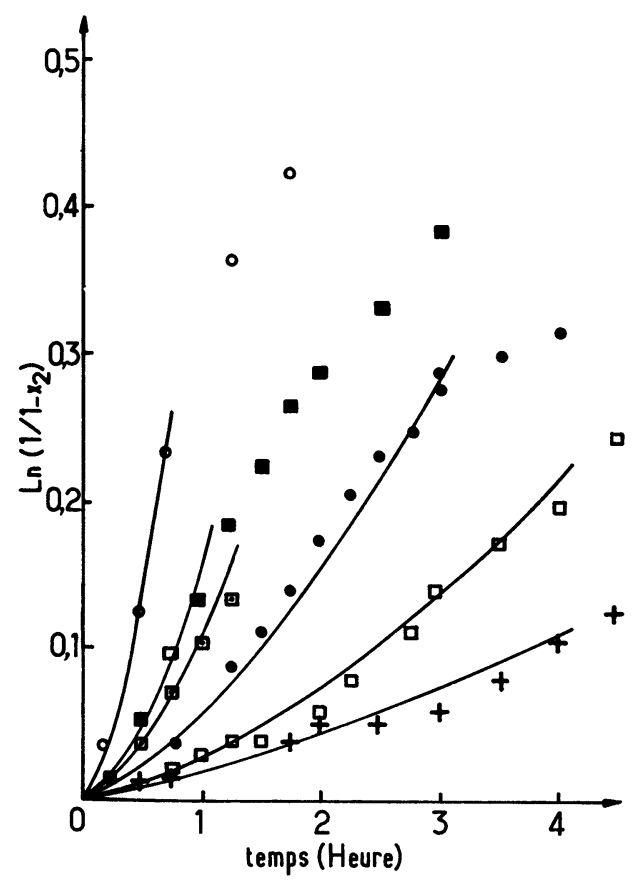

Fig. 5. - Variation de la fraction cristallisée dans les échantillons de la série Ge IV pendant des recuits isothermiques à : - $455^{\circ} \mathrm{C} ; \bigcirc 465^{\circ} \mathrm{C} ; \square 450^{\circ} \mathrm{C} ; \bigcirc 422^{\circ} \mathrm{C} ; \square 415^{\circ} \mathrm{C}$; $+408^{\circ} \mathrm{C}$.

à la taille et à la concentration des cristallites une fois l'échantillon porté à la température où la cinétique est étudiée). Les valeurs de $v_{\mathrm{g}}$ prises pour faire le calcul sont celles données par la figure 1 . Les résultats sont

\section{TABleaU II}

$\begin{array}{lrrrrrr}G_{\mathrm{e}} \mathrm{IV} & 2 & 3 & 4 & 8 & 9 & 10 \\ T(\mathrm{~K}) & 728 & 738 & 723 & 695 & 688 & 681 \\ v_{\mathrm{g}}\left(\AA \mathrm{s}^{-1}\right) & 12 & 17 & 11 & 4 & 3,2 & 2,9 \\ a(\mu \mathrm{m}) & 0,8 & 2 & 2,5 & 2,5 & 2,2 & 3,9 \\ p\left(\mathrm{~cm}^{-2}\right) \times 10^{4} & 1,7 & 2,2 & 1,3 & 3 & 2 & 1\end{array}$

donnés dans le tableau II. Les valeurs de $p$ et a ainsi obtenues sont en bon accord avec des observations par microscope électronique [2].

\section{Bibliographie}

[1] Germain, P., Squelafd, S., Bourgoin, J. et Gheorghin, A., J. Non-Crystall. Solids 1976, à paraître.

[2] Germain, P., Squelard, S., Bourgoin, J. et Gheorghin, A., J. Appl. Phys., 1976, à paraître.

[3] Landauer, R., J. Appl. Phys. 23 (1952) 779.

[4] Barna, A., Barna, P. B. et Pocza, J. F., J. Non-Crystall. Solids 8 (1972) 36. 